Research in Astron. Astrophys. 2011 Vol. 11 No. XX, 000-000

http://www.raa-journal.org http://www.iop.org/journals/raa

$\boldsymbol{R}$ esearch in

Astronomy and

Astrophysics

\title{
Testing the Distance-Duality Relation with a Combination of Cosmological Distance Observations
}

\author{
Shuo Cao ${ }^{1}$ and Nan Liang ${ }^{1,2}$ \\ ${ }^{1}$ Department of Astronomy, Beijing Normal University, Beijing 100875, China; liangn@ bnu.edu.cn \\ 2 Center for High Energy Physics, Peking University, Beijing 100871, China \\ Received [year] [month] [day]; accepted [year] [month] [day]
}

\begin{abstract}
In this paper, we propose an accurate test of the distance-duality (DD) relation, $\eta=D_{L}(z)(1+z)^{-2} / D_{A}(z)=1$ (where $D_{L}$ and $D_{A}$ are the luminosity distances and angular diameter distances, respectively), with a combination of cosmological observational data of Type Ia Supernave (SNe Ia) from Union2 set and the galaxy cluster sample under an assumption of spherical model. In order to avoid bias brought by redshift incoincidence between observational data and to consider redshift error bars of both clusters and $\mathrm{SNe}$ Ia in analysis, we carefully choose the SNe Ia points which have the minimum acceptable redshift difference of the galaxy cluster sample $\left(|\Delta z|_{\min }=\sigma_{z, \mathrm{SN}}+\sigma_{z, \text { cluster }}\right)$. By assuming $\eta$ a constant and functions of the redshift parameterized by six different expressions, we find that there exists no conceivable evidence for variations in the DD relation concerning with observational data, since it is well satisfied within $1 \sigma$ confidence level for most cases. Further considering different values of $\Delta z$ in constraining, we also find that the choosing of $\Delta z$ may play an important role in this model-independent test of the distance-duality relation for the spherical sample of galaxy clusters.
\end{abstract}

Key words: distance scale — galaxies: clusters: general — supernovae: general

\section{INTRODUCTION}

Distance-duality relation, also known as the Etherington's reciprocity relation (Etherington, 1933), is of fundamental importance in cosmology, which relates the luminosity distance $\left(\mathrm{LD}, D_{L}\right)$ with the angular diameter distance (ADD, $D_{A}$ ) by means of the following expression,

$$
\eta=\frac{D_{L}}{D_{A}}(1+z)^{-2}=1 .
$$

We notice that the DD relation is completely general, valid for all cosmological models based on Riemannian geometry, being dependent neither on Einstein field equations nor the nature of matterenergy content. It only requires that source and observer are connected by null geodesics in a Riemannian spacetime and that the number of photons are conserved. This equation plays an essential role in modern cosmology (Csaki et al., 2002), ranging from gravitational lensing studies (Schneider et al., 1992; Fu et al., 2008) to analyzes from galaxy clusters observations (Lima et al., 2003; Cunha et al., 2007), as well as to the plethora of cosmic consequences involving primary and secondary temperature anisotropies of the cosmic microwave background (CMB) observations (Komatsu et al., 2010). 
Up to now, diverse astrophysical mechanisms such as gravitational lensing and dust extinction have been proved to be capable to cause obvious deviation from the distance duality and testing this equality with high accuracy can also provide a powerful probe of exotic physics (Bassett \& Kunz, 2004a b; Corasaniti, 2006). Therefore, it is rewarding to explore the distance-duality relation to test the validity of photon conservation etc. On the side of the observational data, if one is able to find cosmological sources whose intrinsic luminosities are known (standard candles) as well as their intrinsic sizes (standard rulers), one can determine both $D_{L}$ and $D_{A}$, and after measuring the common redshifts, to test directly the above Etherington's result. The possibility of using the Sunyaev-Zeldovich effect (SZE) together with X-ray emission of galaxy clusters to measure angular distances was suggested soon after the SZE was found (Silk \& White, 1978). Using jointly, an independent method to determine distance scales and thus to measure the value of the Hubble constant has been provided (Silk \& White, 1978; Birkinshaw et al., 1991; Inagashi et al., 1995; Nozawa et al., 2006). By using an isothermal spherical model for which the hydrostatic equilibrium model and spherical symmetry assumed, Reese et al. (2002) selected 18 galaxy cluster sample and Bonamente et al. (2006) obtained 38 ADD galaxy clusters sample. De Filippis et al. (2005) have corrected the samples by using an isothermal elliptical model to get 25 ADDs of galaxy clusters. Uzan et al. (2004) considered 18 ADDs sample (Reese et al., 2002) to test the DD relation by assuming the $\Lambda$ CDM model via the technique, $D_{A}^{\text {cluster }}(z)=D_{A}^{\Lambda \text { CDM }}(z) \eta^{2}(z)$. They showed that no violation of the DD relation is only marginally consistent. Using the DD relation for astrophysical research can be found in many works, e. g., Bassett \& Kunz (2004b); More et al. (2009); Avgoustidis et al. (2010); Holanda, Lima \& Ribeiro (2011a); Cao \& Zhu (2011).

In order to test the DD relation in a model-independent way, one should use measurements of $D_{L}$ from cosmological observations directly. The first direct evidence for cosmic acceleration came from Type Ia Supernave (SNe Ia) (Riess, 1998; Perlmutter et al., 1999), which have provided the strongest constraints on the cosmological parameters (Riess et al., 2004, 2007; Astier et al., 2006; Wood-Vasey et al., 2007; Kowalski et al., 2008). De Bernardis et al. (2006) divided the weighted average of galaxy clusters (Bonamente et al., 2006) and SNe Ia (Riess et al., 2004) in redshift bins and concluded that the validity of $\eta=1$ is consistent at $68.3 \%(1 \sigma)$ CL. Recently, Holanda. Lima \& Ribeiro (2010) tested the DD relation with ADD samples and the Constitution set of SNe Ia data (Hicken et al,, 2009). In order to avoid the corresponding bias of redshift differences, a selection criteria $(\Delta z=$ $\left|z_{\mathrm{SN}}-z_{\text {cluter }}\right| \leq 0.005$ ) for a given pair of data set are used. By using two parameterizations of $\eta$ parameter, they found that the DD relation are marginally compatible within $2 \sigma$ CL with the elliptical model sample (De Filippis et al., 2005), and a strong violation $(>3 \sigma)$ of the DD relation with the spherical model sample (Bonamente et al., 2006). However, Li, Wu, \& Yu (2011) found that, by removing more data points of galaxy clusters samples according to the selection criteria, the DD relation can be accommodated at $1 \sigma \mathrm{CL}$ for the elliptical model, and at $3 \sigma \mathrm{CL}$ for the spherical model. Nair et al. (2011) discussed the validity of the DD relation with observational data and ruled out some of the parameterizations significantly.

It is obvious that the difference of redshifts between galaxy clusters and SNe Ia may cause obvious deviation in testing the DD relation. In principle, the only strict criterion to form a given pair is that galaxy clusters and SNe Ia should be at the same redshift. Liang, Cao \& Zhu (2011) have found that the DD relation is satisfied at $1 \sigma \mathrm{CL}$ with the corrected $D_{L}$ located at the same redshift of the corresponding 38 spherical galaxy cluster sample, which are obtained by interpolating from the nearby $\mathrm{SNe}$ Ia of the Union2 set. It should be noted that the redshifts of observations are not determined with infinite accuracy, and there is no point to decrease $\Delta z$ below the total $1 \sigma$ error of observational redshifts $\sigma_{z, \text { tot }}=\sigma_{z, \mathrm{SN}}+\sigma_{z, \text { cluster }}$. Therefore, the finite errors of both clusters and SNe Ia should be taken into account in the analysis. In this paper, we consider redshift error bars of both clusters and SNe Ia in analysis of avoiding bias from redshift differences between observational data to test the DD relation. In practice, $\sigma_{z \text {,tot }}$ is not smaller than 0.002 , therefore it is not appropriate to use a smaller window constraint. For the total 38 data pairs with the spherical sample of galaxy clusters and the Union2 set, we find that differences of redshifts between total 38 data pairs are very small $(\Delta z \leq 0.005)$, and there are 33 pairs for the minimum selection criteria $|\Delta z|_{\min }=\sigma_{z \text {,tot. }}$. Thus we choose the SNe Ia points which have the minimum acceptable redshift difference of the galaxy cluster sample $\Delta z \leq 0.002$. This 
criteria serves a much more stringent one compared with $\Delta z \leq 0.005$ (Holanda. Lima \& Ribeiro, 2010; $\mathrm{Li}, \mathrm{Wu}, \& \mathrm{Yu}, 2011$ ), therefore the accuracy and reliability of our test should be improved. We also find that the choosing of $\Delta z$ may play an important role in this model-independent test.

This paper is organized as follows. In Section 2, we introduce seven parametrizations for the DD relations applied in this work. In Section 3, we present a combined data given by the latest released Union $2 \mathrm{SNe}$ Ia data as well as the 38 galaxy cluster samples under an assumption of spherical model. In Section 4 we briefly describe the analysis method and show results for constraining parameters of the DD relation. Finally, we summarize conclusions in Section 5 ,

\section{DD RELATION PARAMETRIZATIONS}

Regarding the parametrization of the DD relation, a model independent test has been extensively discussed in the above quoted papers (De Bernardis et al., 2006; Holanda, Lima \& Ribeiro, 2010, 2011a, b; Li, Wu, \& Yu, 2011; Nair et al., 2011; Liang, Cao \& Zhu, 2011; Meng, Zhang \& Zhan, 2011). De Bernardis et al. (2006) considered $\eta$ a constant with no relation to the redshift

$$
\text { I. } \eta=\eta_{0},
$$

where $\eta_{0}$ is a constant to be constrained by observational data. In general, $\eta$ can be treated as parameterized functions of the redshift, $\eta(z)$, which are clearly inspired on similar expressions for $w(z)$, the equation of state in time-varying dark energy models (see, for instance, Chevallier \& Polarski (2001); Linder (2003); Cunha et al. (2007); Silva, Alcaniz \& Lima (2007)). Recently, Holanda, Lima \& Ribeiro (2010, 2011a) used two one-parameter expressions, namely,

$$
\begin{aligned}
& \text { II. } \eta(z)=1+\eta_{a} z, \\
& \text { III. } \eta(z)=1+\eta_{a} z /(1+z)
\end{aligned}
$$

In this work, we also use another general parametric representations for a possible redshift dependence of the distance duality expression including three two-parameter parameterizations ( $\mathrm{Li}, \mathrm{Wu}, \& \mathrm{Yu}$, 2011; Nair et al,, 2011; Liang. Cao \& Zhu, 2011; Meng. Zhang \& Zhan, 2011)

$$
\begin{aligned}
& \text { IV. } \eta(z)=1+\eta_{a} \ln (1+z), \\
& \text { V. } \eta(z)=\eta_{0}+\eta_{a} z, \\
& \text { VI. } \eta(z)=\eta_{0}+\eta_{a} z /(1+z), \\
& \text { VII. } \eta(z)=\eta_{0}+\eta_{a} \ln (1+z) .
\end{aligned}
$$

\section{GALAXY CLUSTERS AND SUPERNOVAE IA SAMPLES}

In this work, we consider the sample of ADD from galaxy clusters obtained by combining their SZE and X-ray surface brightness observations sample (Bonamente et al., 2006). Under an assumption of spherical model, the cluster plasma and dark matter distributions were analyzed assuming hydrostatic equilibrium model and spherical symmetry, thereby accounting for radial variations in density, temperature and abundance. Recently, the Supernova Cosmology Project (SCP) collaboration have released their Union2 compilation which consists of 557 SNe Ia (Amanullah et al., 2010), which is the largest published and spectroscopically confirmed SNe Ia sample to date.

For a given $D_{A}^{\text {cluster }}$ data point, theoretically, we should select an associated SNe Ia data point $D_{L}^{\mathrm{SN}}$ at the same redshift to obtain an $\eta_{\text {obs }}$. In order to avoid any bias of redshift differences between SNe Ia and galaxy clusters and to consider redshift error bars of both clusters and SNe Ia in analysis, we should determine the value of $\sigma_{z, \text { tot }}=\sigma_{z, \mathrm{SN}}+\sigma_{z, \text { cluster }}$ for the combination of observational data pairs. For the observations of SNe Ia, the peculiar velocity uncertainty is set at $400 \mathrm{kms}^{-1}$ (Wood-Vasev et al., 2007) (or $300 \mathrm{kms}^{-1}$, Kowalski et al. (2008)) and the redshift uncertainty is $\sigma_{z, \mathrm{SN}}=0.001$ (Hicken et al., 2009). For the observations of galaxy clusters, the rms one-dimensional cluster peculiar velocity uncertainty is set at $256_{-75}^{+106} \mathrm{kms}^{-1}$, which corresponds to the three-dimensional rms velocity $459_{-130}^{+184} \mathrm{kms}^{-1}$ 
Watkins, 1997) (or $341 \pm 93 \mathrm{kms}^{-1}$ for the rms one-dimensional cluster peculiar velocity, which corresponds to the three-dimensional rms velocity $591 \pm 161 \mathrm{kms}^{-1}$, Dale et al. (1999)) and the redshift uncertainty is $\sigma_{z \text {,cluster }}=0.001$. Therefore, $\Delta z=\sigma_{z \text {,tot }}=0.002$ is considered in our work. Obviously, this strict choice with $\Delta z=0.002$ may helpfully ease the systematic errors brought by redshift inconsistence between SNe Ia and galaxy clusters. Therefore, we obtain a sub-sample of SNe Ia from the Union2 data set whose redshifts coincide with the ones appearing in the galaxy cluster sample under this criterion. We then bin the SNe data in the redshift bins of the corresponding spherical galaxy cluster sample to obtain 33 data pairs in our test. Assuming that $\mu_{i}$ represents the ith appropriate SNe Ia distance modulus data (within the $|\Delta z|<0.002$ redshift range) with $\sigma_{\mu_{i}}$ denoting its reported observational uncertainty, in light of standard data reduction framework by Bevington \& Robinson $(2003$, Chap. 4), we obtain

$$
\begin{aligned}
& \bar{\mu}=\frac{\sum\left(\mu_{i} / \sigma_{\mu_{i}}^{2}\right)}{\sum 1 / \sigma_{\mu_{i}}^{2}}, \\
& \sigma_{\bar{\mu}}^{2}=\frac{1}{\sum 1 / \sigma_{\mu_{i}}^{2}},
\end{aligned}
$$

where $\bar{\mu}$ stands for the weighted mean distance modulus at the corresponding galaxy cluster redshift and $\sigma_{\bar{\mu}}$ serves as its uncertainty.

It must be emphasized that, if a redshift-dependent expression for the DD relation is considered, the SZE+X-ray surface brightness observation technique gives $D_{A}^{\text {cluster }}(z)=D_{A}(z) \eta^{2}(z)$ (Sunyaev \& Zel'dovich, 1972; Cavaliere \& Fusco-Fermiano, 1978). So, we must replace $D_{A}(z)$ with $D_{A}^{\text {cluster }}(z) \eta^{-2}$ when we try to test the reciprocity relation consistently with the SZE + X-ray observations from galaxy clusters. Thus, the observed $\eta_{\mathrm{obs}}(z)$ is determined by the following expression:

$$
\eta_{\mathrm{obs}}(z)=D_{A}^{\text {cluster }}(z)(1+z)^{2} / D_{L}(z) \text {. }
$$

It should be noted that the data points of the compiled Union2 SNe Ia are given in terms of the distance modulus, which could reduce to

$$
D_{L}(z)=10^{\mu(z) / 5-5} .
$$

Accordingly, the uncertainty of the luminosity distance could be expressed in term of the distance modulus uncertainty $\sigma_{D_{L}(z)}=\ln 10 / 5 \times 10^{(\mu / 5-5)} \sigma_{\mu(z)}$.

\section{ANALYSIS AND RESULTS}

In this section, we estimate the $\eta_{0}$ and $\eta_{a}$ parameters in seven parametrizations listed in Sec. 2] To estimate the model parameters of a given parameterized form, we use the minimum $\chi^{2}$ estimator following standard route

$$
\chi^{2}(z ; \mathbf{p})=\sum_{z} \frac{\left[\eta_{\mathrm{th}}(z ; \mathbf{p})-\eta_{\mathrm{obs}}(z)\right]^{2}}{\sigma_{\eta_{\mathrm{obs}}}^{2}},
$$

where $\eta_{\mathrm{th}}$ represents the theoretical value of $\eta$ parameter with the parameter set $\mathbf{p}$, and $\eta_{\mathrm{obs}}$ associated with the observational technique with the error of $\sigma_{\eta_{\mathrm{obs}}}$, which comes from the statical contributions and systematic uncertainties of the galaxy clusters and SNe Ia, as well as the redshifts

$$
\sigma_{\eta_{\mathrm{obs}}}=\sigma_{D_{A}}^{\text {cluster }}(1+z)^{2} / D_{L}-D_{A}^{\text {cluster }} \sigma_{D_{L}}(1+z)^{2} / D_{L}^{2}+2 D_{A}^{\text {cluster }} \sigma_{z}(1+z) / D_{L} .
$$

For the one-parameter models, one should expect the likelihood of $\eta_{0}$ or $\eta_{a}$ peaks at $\eta_{0}=1$ or $\eta_{a}=0$ ( $\Delta \chi^{2}$ minimizes at $\eta_{0}=1$ or $\eta_{a}=0$ ), in order to satisfy the DD relation. As to the two-parameter models, one should expect $\eta_{0}=1$ and $\eta_{a}=0$ to be the best-fit parameters in the confidence contours, if it consists with photon conservation and no visible violation of the DD relation.

In Fig. 1 (Left), we plot the likelihood distribution function in the $\eta_{0}-\Delta \chi^{2}$ plane and obtain $\eta_{0}=0.97_{-0.06}^{+0.05}$ at $1 \sigma$, which is in good qualitative accord with previous analyses $\left(\eta_{0}=1.01_{-0.07}^{+0.07}\right)$ (De Bernardis et al., 2006). In Fig. 1 (Right), we show the likelihood distribution function from three 

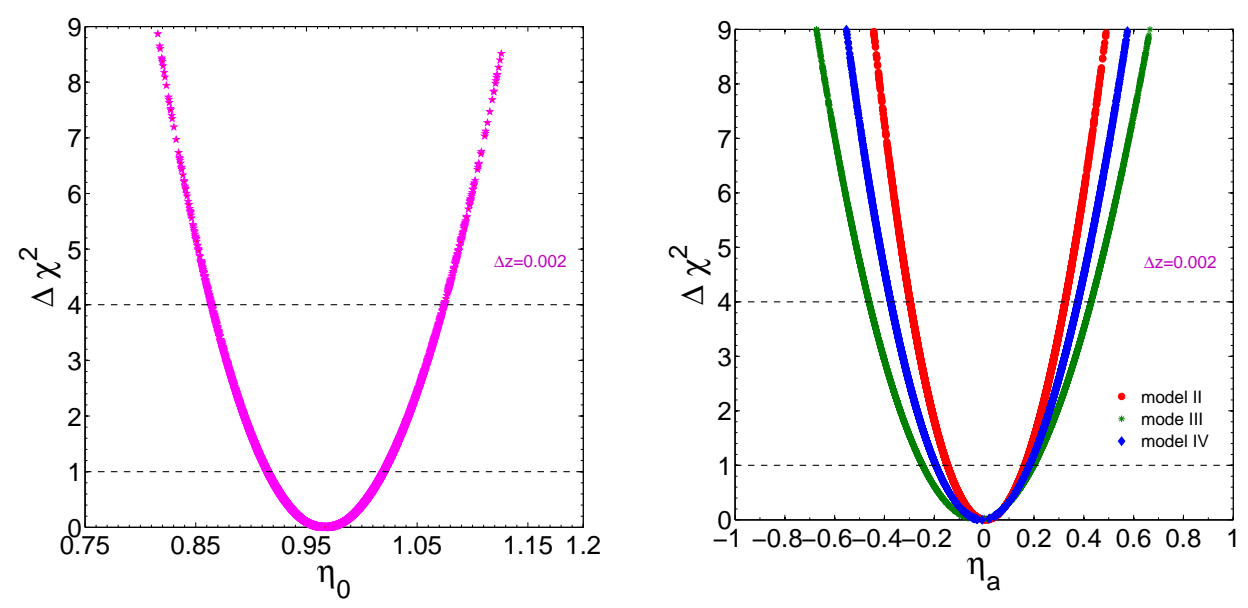

Fig. 1 The likelihood distribution function with the 33 ADDs of galaxy clusters and the luminosity distances of the Union2 set for one-parameter forms in the $\eta_{0}-\Delta \chi^{2}$ planes (for model I) and $\eta_{a}-\Delta \chi^{2}$ planes (for model II-IV) with $\Delta z=0.002$.

one-parameter forms of the redshift: II. $\eta(z)=1+\eta_{a} z$; III. $\eta(z)=1+\eta_{a} z /(1+z)$; and IV. $\eta(z)=$ $1+\eta_{a} \ln (1+z)$. The best-fit values at $1 \sigma \mathrm{CL}$ are $\eta_{a}=-0.01_{-0.16}^{+0.15}$ for model I., $\eta_{a}=-0.01_{-0.24}^{+0.21}$ for model II., and $\eta_{a}=-0.01_{-010}^{+0.22}$ for model III., respectively, which are different from those obtained in Holanda, Lima \& Ribeiro (2010), where the DD relation is ruled out at $3 \sigma \mathrm{CL}$, and those obtained in $\mathrm{Li}$. Wu, \& Yu (2011), where the DD relation is accommodated at $3 \sigma \mathrm{CL}$ for the spherical model. Fitting results from one-parameter forms with the ADDs of galaxy clusters and the luminosity distances of the Union2 set with $\Delta z=\sigma_{z \text {,tot }}=0.002$ are summarized in Table 1 .

\begin{tabular}{|l|c|c|}
\hline Parameterization $(\Delta z=0.002)$ & $\eta_{0}$ & $\eta_{a}$ \\
\hline I. $\eta_{0}$ & $0.97_{-0.06}^{+0.05}$ & 0 \\
\hline II. $1+\eta_{a} z$ & 1 & $-0.01_{-0.16}^{+0.15}$ \\
\hline III. $1+\eta_{a} \frac{z}{1+z}$ & 1 & $-0.01_{-0.24}^{+0.21}$ \\
\hline IV. $1+\eta_{a} \ln (1+z)$ & 1 & $-0.01_{-0.19}^{+0.22}$ \\
\hline V. $\eta_{0}+\eta_{a} z$ & $0.84_{-0.17}^{+0.17}$ & $0.43_{-0.49}^{+0.49}$ \\
\hline VI. $\eta_{0}+\eta_{a} \frac{z}{1+z}$ & $0.74_{-0.22}^{+0.23}$ & $1.02_{-0.85}^{+0.84}$ \\
\hline VII. $\eta_{0}+\eta_{a} \ln (1+z)$ & $0.82_{-0.19}^{+0.20}$ & $0.57_{-0.67}^{+0.68}$ \\
\hline
\end{tabular}

Table 1 Summary of the results for seven parameterizations at $1 \sigma$ confidence level with $\Delta z=0.002$.

The above analyses are based on the assumption that the redshift-independent model parameter is a constant $\eta_{0}=1$. Now we take it as a varying parameter to examine the DD relation by assuming more general expressions: V. $\eta(z)=\eta_{0}+\eta_{a} z$; VI. $\eta(z)=\eta_{0}+\eta_{a} z /(1+z)$; VII. $\eta(z)=\eta_{0}+\eta_{a} \ln (1+z)$. Fitting results from two-parameter forms with the ADDs of galaxy clusters and the luminosity distances of the Union2 set with $\Delta z=\sigma_{z \text {,tot }}=0.002$ are shown in Fig. 2 and summarized in Table 1 Our results suggest that there is no violation of the DD relation for two-parameter parameterizations at $1 \sigma$ CL for model V and VII, and at $2 \sigma \mathrm{CL}$ for model VI, which are more stringent than those obtained in 

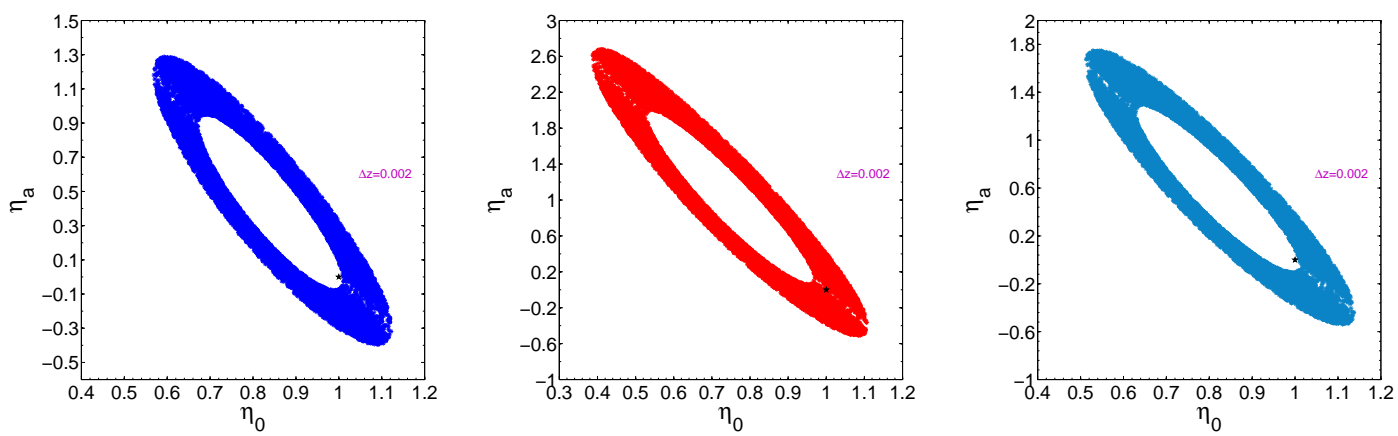

Fig. 2 Likelihood contours with the 33 ADDs of galaxy clusters and the luminosity distances of the Union 2 set at 1 and $2 \sigma$ CL for two-parameter forms in the $\eta_{0}-\eta_{a}$ plane [Left: for $\eta(z)=\eta_{0}+\eta_{1} z$; Middle: for $\eta(z)=\eta_{0}+\eta_{a} \frac{z}{1+z}$; Right: for $\left.\eta(z)=\eta_{0}+\eta_{a} \ln (1+z)\right]$. The filled stars represent the cases with no violation of the DD relation $\left(\eta_{0}=1\right.$ and $\left.\eta_{a}=0\right)$.

Li, Wu, \& Yu (2011), where the DD relation are consistent at $2 \sigma \mathrm{CL}$ for the spherical sample of galaxy clusters.

From Fig. 1, 2 and Table 11 we can find that the DD relation can be accommodated at $1 \sigma$ CL for the Bonamente et al. sample except model VI. Our results differs from those obtained by Holanda, Lima \& Ribeiro (2010), where the results from the Bonamente et al. sample give a clear violation of the DD relation. However, these results are more stringent than those obtained by Li. Wu, \& Yu (2011), where the DD relation is accommodated at $2-3 \sigma$ CL for the spherical sample of galaxy clusters.

After identifying the constraints on $\eta$ obtained with the minimum acceptable $\Delta z=0.002$, we may consider different values of $\Delta z$ for examining the role of the choosing of $\Delta z$ played in constraints. For the selection criteria of $\Delta z=0.003,0.004,0.005$, there are $35,37,38$ data pairs, respectively. In Fig. 3, we show the corresponding constraints on $\eta_{a}$ for the three one-parameter forms of the redshift: II. $\eta(z)=1+\eta_{a} z$; III. $\eta(z)=1+\eta_{a} z /(1+z)$; and IV. $\eta(z)=1+\eta_{a} \ln (1+z)$. Finally, we plot the $1 \sigma$ error bar of $\eta_{a}$ as a function of $\Delta z=0.002-0.005$ in Fig. 4. For comparison, we also show the results of $\Delta z=0$ with 14 data pairs.

From Fig. 3. 4, we can find that the choosing of $\Delta z$ may play an important role in this modelindependent test, and the results for $\Delta z=0.005$ in our test show the DD relation is ruled out at $2 \sigma \mathrm{CL}$, which are close to those of Holanda. Lima \& Ribeiro (2010) where the DD relation is ruled out at $3 \sigma$ $\mathrm{CL}$, and consistent with those obtained in Li. Wu, \& Yu (2011), where the DD relation are consistent at $3 \sigma$ CL for the spherical sample of galaxy clusters.

\section{CONCLUSIONS}

In this paper, we have discussed a new model-independent cosmological test for the distance-duality relation, $\eta(z)=D_{L}(1+z)^{-2} / D_{A}$. We consider the angular diameter distances from galaxy clusters obtained by using Sunyaev-Zeldovich effect and X-ray surface brightness together with the luminosity distances given a sub-sample of SNe Ia taken from the Union2 data. The key aspect is that SNe Ia is carefully chosen to have the minimum acceptable redshift difference of the galaxy cluster $(\Delta z=$ $\left.\sigma_{z, \text { tot }}=\sigma_{z, \mathrm{SN}}+\sigma_{z \text {,cluster }}\right)$. For the sake of generality, the $\eta$ parameter is also parameterized in seven different forms, namely, four one-parameter models.: (I) $\eta=\eta_{0}$, (II) $\eta=1+\eta_{a} z$, (III) $\eta=1+$ $\eta_{a} z /(1+z)$, (IV) $\eta=1+\eta_{a} \ln (1+z)$ and three two-parameter models: (V) $\eta=\eta_{0}+\eta_{a} z,(\mathrm{VI}) \eta=$ $\eta_{0}+\eta_{a} z /(1+z),(\mathrm{VII}) \eta=\eta_{0}+\eta_{a} \ln (1+z)$. 

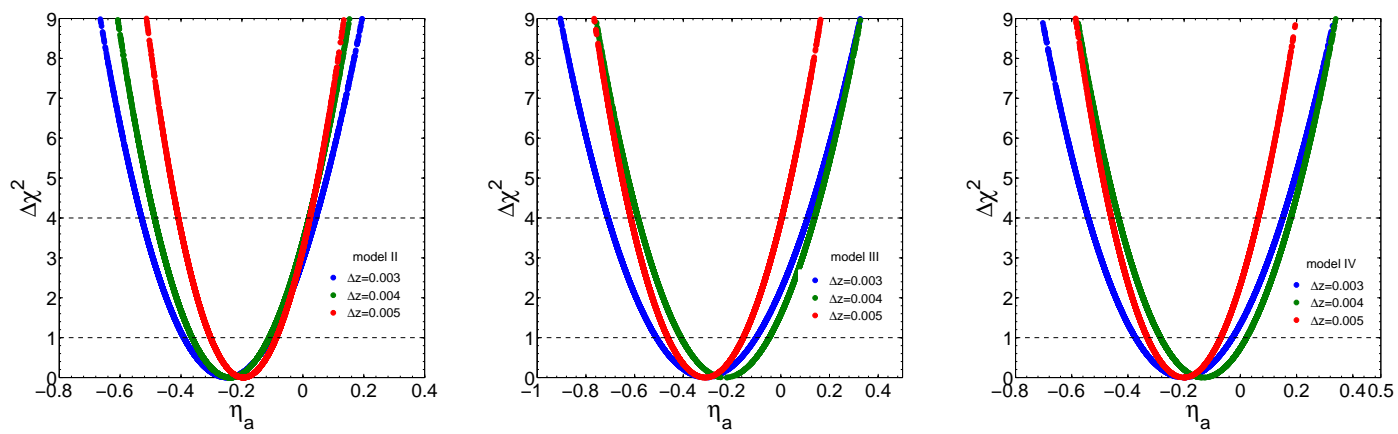

Fig. 3 The likelihood distribution function in the $\eta_{a}-\Delta \chi^{2}$ planes for the three one-parameter forms of the redshift (model II, III, IV) with varying $\Delta z=0.003,0.004,0.005$.

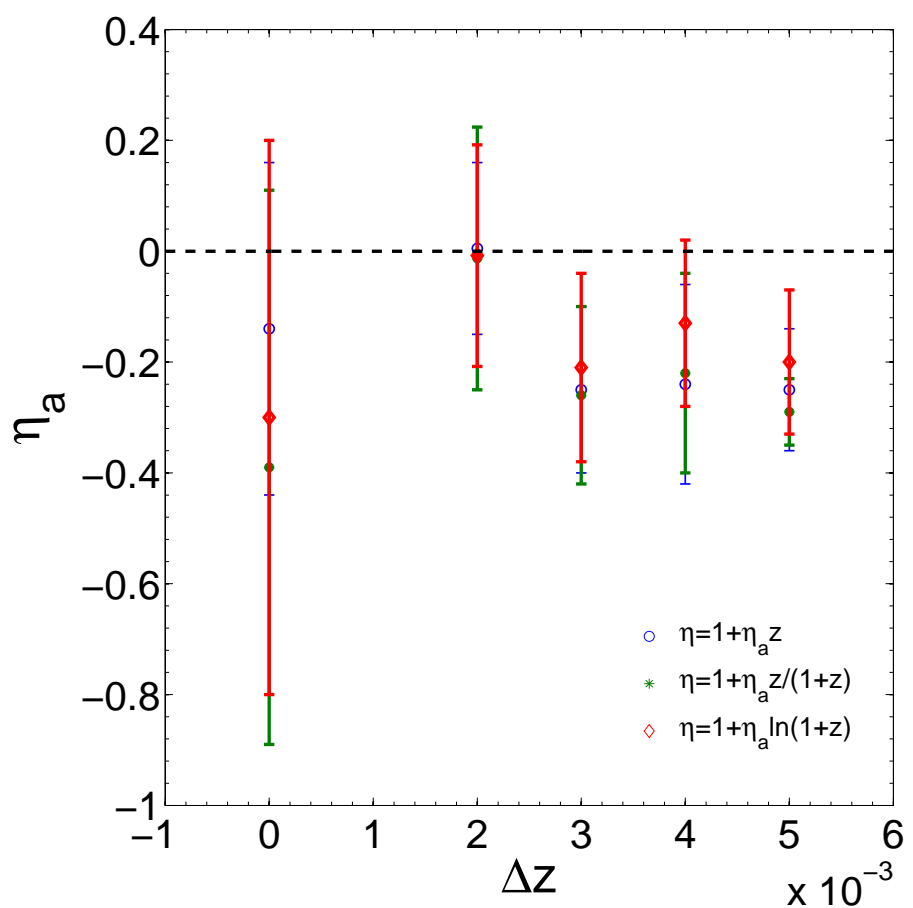

Fig. 4 The $1 \sigma$ error bar of $\eta_{a}$ as a function of $\Delta z=0-0.005$ for the three one-parameter forms of the redshift (model II, III, IV).

By assuming $\eta$ a constant, we obtain $\eta_{0}=0.97_{-0.06}^{+0.05}$ at $1 \sigma$. For the redshift-dependent oneparameter forms of model II, model III, and model IV, we obtain $\eta_{a}=-0.01_{-0.16}^{+0.15}, \eta_{a}=-0.01_{-0.24}^{+0.21}$, and $\eta_{a}=-0.01_{-0.19}^{+0.22}$, respectively, which are well consistent with no violation of the distance-duality relation. We furthermore put forwards three kinds of two-parameter parametrizations corresponding to 
model II, III and IV, respectively. The standard values without any violation of the reciprocity relation $\left(\eta_{0}=1\right.$ and $\left.\eta_{a}=0\right)$ is still included at $68.3 \% \mathrm{CL}(1 \sigma)$ for model V, VII and at 95.8\% CL ( $\left.2 \sigma\right)$ for model VI. It is shown that there is no conceivable evidence for variations of the duality distance relation for the Bonamente et al. sample, since it is marginally satisfied within $1 \sigma \mathrm{CL}$ for most cases, which is different from those obtained by Holanda. Lima \& Ribeiro (2010), where the results from the Bonamente et al. sample give a clear violation of the DD relation, and more stringent than those obtained by Li. Wu, \& Yu (2011). By further considering different values of the redshift difference $\Delta z$, we find that the choosing $\Delta z$ may play an important role in this model-independent cosmological test of the DD relation, and the results for $\Delta z=0.005$ in our test show the DD relation is ruled out at $2 \sigma \mathrm{CL}$, which are close to those of Holanda. Lima \& Ribeiro (2010) where the DD relation is ruled out at $3 \sigma \mathrm{CL}$, and consistent with those obtained in $\mathrm{Li}, \mathrm{Wu}, \& \mathrm{Yu}$ (2011), where the DD relation are consistent at $3 \sigma \mathrm{CL}$ for the spherical sample of galaxy clusters.

It is still interesting to see whether those conclusions may be changed with larger SNe Ia and clusters data in the future, which reinforces the interest in the observational search for more samples of galaxy clusters with smaller statistical and systematic uncertainties, as well as the determination of their angular diameters trough the combination of SZE and X-ray surface brightness.

Acknowledgements We thank Prof. Zong-Hong Zhu, Dr. Yi Zhang, as well as Heng Yu, XiaoLong Gong, Hao Wang, Xing-Jing Zhu, Yan Dai, Yu Pan, Fang Huang, Jing Ming, Kai Liao for discussions. This work was supported by the National Natural Science Foundation of China under the Distinguished Young Scholar Grant 10825313 and Grant 11073005, the Ministry of Science and Technology national basic science Program (Project 973) under Grant No.2007CB815401, the Fundamental Research Funds for the Central Universities and Scientific Research Foundation of Beijing Normal University.

\section{References}

Amanullah, R., et al. 2010, ApJ, 716, 712

Astier, P., et al. 2006, A\&A, 447, 31

Avgoustidis, A. et al. 2010, JCAP, 10, 024

Bassett, B. A., \& Kunz, M. 2004a, ApJ. 607, 661

Bassett, B. A., \& Kunz, M. 2004b, PRD, 69, 101305

Bevington, P. R., \& Robinson, D. K. 2003, Data reduction and error analysis for the physical sciences, 3rd ed., by Philip R. Bevington, and Keith D. Robinson. Boston, MA: McGraw-Hill, ISBN 0-07247227-8, 2003

Birkinshaw, M., Hugues, J. P., \& Arnaud, K. A., 1991, ApJ, 379, 466.

Bonamente, M., et al. 2006, ApJ, 647, 25

Cao, S., \& Zhu, Z.-H. 2011, ScChG in press

Cavaliere, A., \& Fusco-Fermiano, R. 1978, A\& A, 667, 70

Chevallier, M. \& Polarski, D., 2001, IJMPD, 10, 213

Corasaniti, P. S. 2006, MNRAS, 372, 191

Csaki, C., Kaloper, N. \& Terning, J. 2002, PRL, 88, 161302

Cunha, J. V., Marassi, L., \& Lima, J. A. S. 2007, MNRAS, 379, L1

Dale, D. A., et al. 1999, AJ, 118, 1489

De Bernardis, F., Giusarma, E., \& Melchiorri, A. 2006, IJMPD, 15, 759

De Filippis, E., SerenoM, M., Bautz, W., Longo, G. 2005, ApJ, 625, 108

Etherington, I. M. H. 1933, Phil. Mag. 15, 761; reprinted in 2007, Gen. Rel. Grav. 39, 1055

Fu, L., et al. 2008, A\&A, 479, 9

Hicken, M., et al. 2009, ApJ, 700, 1097

Holanda, R. F. L., Lima, J. A. S., \& Ribeiro, M. B., 2010, ApJ, 722, L233

Holanda, R. F. L., Lima, J. A. S., \& Ribeiro, M. B., 2011, A\&A, 528, 14

Holanda, R. F. L., Lima, J. A. S., \& Ribeiro, M. B., arXiv:1104.3753

Inagashi, Y., Suginohara, T., \& Suto, Y. 1995, Publ. Astron. Soc. Japan, 47, 411 
Komatsu, E., et al. [WMAP collaboration], 2010, arXiv:1001.4538

Kowalski, M., et al. 2008, ApJ, 686, 749

Lima, J. A. S., Cunha, J. V., \& Alcaniz, J. S. 2003, PRD, 68, 023510

Linder E. V. 2003, PRL, 90, 091301

Li, Z. X., Wu, P. X., \& Yu, H. W. 2011, ApJ, 729, L14

Liang, N., Cao, S., \& Zhu, Z.-H. 2011, arXiv:1104.2497

Meng, X.-L., Zhang, T.-J., \& Zhan, H. 2011, arXiv:1104.2833

More, S., Bovy, J., \& Hogg, D. W. 2009, ApJ, 696, 1727

Nair, R., Jhingan, S., \& Jain, D. 2011 [arXiv:1102.1065]

Nozawa, S., Itoh, N., Suda, Y., \& Ohhata, Y. 2006, Nuovo Cimento B, 121, 487 [arXiv:0507466]

Perlmutter, S., et al. 1999, ApJ, 517, 565

Reese, E. D., et al. 2002, ApJ, 581, 53

Riess, A. G., et al. 1998, AJ, 116, 1009

Riess, A. G., et al. [Supernova Search Team Collaboration], 2004, ApJ, 607, 665

Riess, A. G., et al. 2007, ApJ, 659, 98

Schneider, P., Ehlers, J., \& Falco, E. E. 1992, Gravitational Lenses (Springer-Verlag, Berlin)

Silk, J., \& White, D. M., 1978, ApJL, 226, L103

Silva, R., Alcaniz, J. S., \& Lima, J. A. S. 2007, IJMPD, 16, 469

Sunyaev, R. A., \& Zel'dovich, Y. B. 1972, Comm. Astrophys. Space Phys., 4, 173

Uzan, J. P., Aghanim, N., \& Mellier, Y. 2004, PRD, 70, 083533

Watkins, R. 1997, MNRAS, 292, L59

Wood-Vasey, W. M., et al. 2007, ApJ, 666, 694 